\title{
Epidemiological and clinical course of 483 patients with COVID-19 in Wuhan, China: a single-center, retrospective study from the mobile cabin hospital
}

\author{
Bo Wang ${ }^{1} \cdot$ Zhixian Wang $^{2} \cdot$ Jianping Zhao ${ }^{3} \cdot$ Xiaoyong Zeng $^{2} \cdot$ Mingfu Wu $^{1} \cdot$ Shixuan Wang ${ }^{1} \cdot$ Tiejun Wang $^{4}$
}

Received: 26 March 2020 / Accepted: 10 May 2020 / Published online: 18 July 2020

(C) The Author(s) 2020

\begin{abstract}
During the COVID-19 outbreak, the mobile cabin hospital has effectively isolated and treated patients diagnosed as mildmoderate disease. However, a detailed clinical course has not been well described. We included 483 patients who were isolated and treated from Feb 6, 2020, to Feb 15, 2020, including definite outcome (discharge or deterioration). Sixty-two patients were transferred to severe cases, of whom were trasfered to designated hospital for intensive care. By March 9, 2020, all patients were discharged without dead. The mobile cabin hospital provides feasible strategy of isolation of mild-moderate cases and timely intervention during the virus outbreak.
\end{abstract}

Keywords COVID-19 $\cdot$ characteristics $\cdot$ outcomes $\cdot$ mobile cabin hospital

\author{
Abbreviations \\ BMI Body mass index \\ IQR Interquartile range \\ SD Standard deviation \\ COVID-19 Corona virus disease 2019
}

\section{Introduction}

Since December 8, 2019, Wuhan, Hubei, China, has reported several cases of COVID-19. In addition to China, other countries including South Korea, Iran, and Italy also have reported

Bo Wang and Zhixian Wang contributed equally to this work.

Electronic supplementary material The online version of this article (https://doi.org/10.1007/s10096-020-03927-3) contains supplementary material, which is available to authorized users.

Shixuan Wang

shixuanwang@126.com

Tiejun Wang

tiejunwanghp@163.com

1 Department of Obstetrics and Gynecology, Tongji Hospital, Tongji Medical College, Huazhong University of Science and Technology, Wuhan 430030, China cases of COVID-19 infection [1, 2]. According to the "New Coronavirus Infected Pneumonia Diagnosis and Treatment Plan (Trial Version 5)," during the study period [3], severe and critically ill patients are at risk for secondary systemic multiple organ failure, which in turn increases the risk of death. Therefore, it is necessary to treat critically ill patients and also prevent mild-moderate cases from developing into severe cases. The mobile cabin hospital has played an important role in stemming China's outbreak of COVID-19 infection, especially in isolating and treating patients diagnosed as mild-moderate disease. However, information about these patient's characteristics and the outcomes are scarce. Although
2 Department of Urology, Tongji Hospital, Tongji Medical College, Huazhong University of Science and Technology, Wuhan 430030, China

3 Department of Respiratory and Critical Care Medicine, National Clinical Research, Key Laboratory of Pulmonary Diseases of Health Ministry, Tongji Hospital, Tongji Medical College, Huazhong University of Science and Technology, Wuhan 430030, Hubei, China

4 Department of Breast Surgery, Hubei Cancer Hospital, 116 Zhuodaoquan South Road, Wuhan 430079, China 
previous studies reported the clinical characteristics of patients with COVID-19 pneumonia [4-7], limited research focused on the patients who developed from mildmoderate to severe disease, our study mainly analyzed the clinical characteristics of these cases admitted to the mobile cabin hospital (Fig. 1).

\section{Results}

Among the cohort of 483 patients, 62 patients (12.8\%) progressed to severe cases, and 421 patients $(87.2 \%)$ were cured. The median age was 50 years, and $54.9 \%$ of cases were female. Besides, $61.7 \%$ of patients were exposed to the suspected/confirmed patients, and $45.5 \%$ of patients experienced family cluster infection. We found increasing odds of severe cases associated with comorbidities, including primary pulmonary disease $(7.6 \%$ vs. $16.1 \%, p=0.047)$, coronary heart disease $(0.7 \%$ vs. $8.1 \%, p<0.001)$, and abnormal laboratory test of renal $(0.2 \%$ vs. $16.1 \%, p<0.001)$, liver function (1.9\%vs. $19.4 \%, p<0.001)$, renal function $(0.2 \%$ vs. $16.1 \%$, $p<0.001)$, heart function $(1.2 \%$ vs. $19.4 \%, p<0.001)$, and abnormal lymphocyte $(15.2 \%$ vs. $37.1 \%, p<0.001)$ and leukocyte counts $(23.0 \%$ vs. $37.1 \%, p=0.025)$ (Table 1$)$. By March 9, 2020, all patients were as follows: 62 patients who were diagnosed as severe cases were transferred to a designated hospital for intensive care, of whom, 23 refused (alive confirmed), 8 patients were cured and discharged, and 31 patients were still in the designated hospital to continue treatment and recovered.

\section{Discussion}

During the COVID-19 outbreak, the number of confirmed cases has exploded in China. The major challenge is to treat and isolate these patients, as well as reduce severe cases and mortality. The establishment of the mobile cabin hospital has witnessed the classification management effectively. In this study, all patients received a nucleic acid test before admission; after the patients were admitted to the mobile cabin hospital, the treatment was carried out according to the "New Coronavirus Infected Pneumonia Diagnosis and Treatment Plan" [3]. To our knowlegement, this is the largest retrospective cohort study among mild-moderate cases with COVID-19 infection; the clinical course with respect to mild-moderate and severe cases in Wuchang mobile cabin hospital were analyzed in this study.

Our results showed that there was no significant difference in fever between mild-moderate and severe cases, of whom $421(87.2 \%)$ patients were not admitted to the ICU, and 263 $(62.5 \%)$ patients were identified as having a fever but
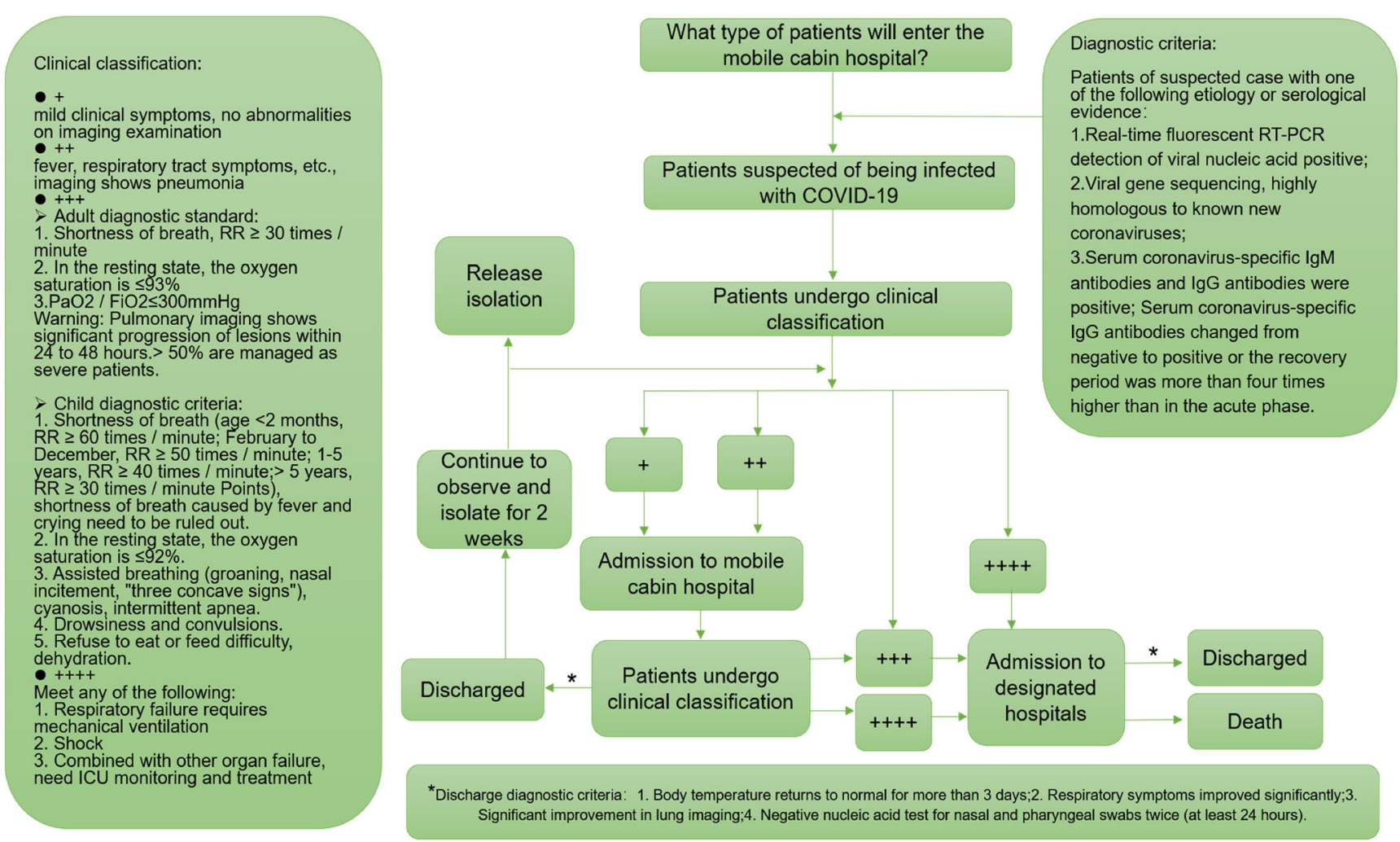

Fig. 1 The flow chart of consultation for patients infected with COVID-19 
Table 1 Clinical characteristics of patients with coronavirus disease 2019

\begin{tabular}{|c|c|c|c|c|}
\hline & Overall & Mild-moderate cases $(+/++)$ & Severe cases $(+++/++++)$ & $p^{\mathrm{a}}$ value \\
\hline & $(n=483)$ & $(n=421)$ & $(n=62)$ & \\
\hline Time from symptom to admission & & & & 0.585 \\
\hline Mean (SD, min, max) & $5.86(5.23,0.00,30.0)$ & $5.90(5.27,0.00,30.0)$ & $5.52(4.91,0.00,18.0)$ & \\
\hline Median (IQR) & $4.00(2.00,8.00)$ & $4.00(2.00,8.00)$ & $3.00(2.00,9.00)$ & \\
\hline Time from admission to cure/severe illness & & & & $<0.001$ \\
\hline Mean (SD, min, max) & $12.2(4.71,1.00,23.0)$ & $12.5(4.51,1.00,23.0)$ & $9.74(5.32,1.00,23.0)$ & \\
\hline Median (IQR) & $12.0(9.00,15.0)$ & $13.0(9.00,16.0)$ & $9.00(5.25,13.8)$ & \\
\hline Time from symptom to cure/severe illness & & & & 0.002 \\
\hline Mean (SD, min, max) & $18.0(7.41,1.00,50.0)$ & $18.4(7.35,1.00,50.0)$ & $15.3(7.32,1.00,50.0)$ & \\
\hline Median (IQR) & $17.0(13.0,23.0)$ & $17.0(13.0,23.0)$ & $14.5(9.25,21.0)$ & \\
\hline Demographic characteristics & & & & 0.830 \\
\hline \multicolumn{5}{|l|}{ Age } \\
\hline Mean (SD, min, max) & $48.4(12.4,11.0,83.0)$ & $48.5(12.4,11.0,72.0)$ & $48.1(12.9,22.0,83.0)$ & \\
\hline Median (IQR) & $50.0(39.0,58.0)$ & $50.0(39.0,58.0)$ & $48.5(37.3,58.8)$ & \\
\hline Age distribution & & & & 0.360 \\
\hline$(\sim 50]$ & $227(47.0 \%)$ & $194(46.1 \%)$ & $33(53.2 \%)$ & \\
\hline$(50 \sim$ & $256(53.0 \%)$ & $227(53.9 \%)$ & $29(46.8 \%)$ & \\
\hline Sex & & & & 0.341 \\
\hline Female & $265(54.9 \%)$ & $227(53.9 \%)$ & $38(61.3 \%)$ & \\
\hline Male & $218(45.1 \%)$ & $194(46.1 \%)$ & $24(38.7 \%)$ & \\
\hline BMI & & & & 0.738 \\
\hline Mean (SD, min, max) & $23.3(3.15,15.0,37.8)$ & $23.3(3.15,15.0,37.8)$ & $23.4(3.15,16.5,31.3)$ & \\
\hline Median (IQR) & $22.9(21.4,25.4)$ & $22.9(21.4,25.4)$ & $23.1(21.9,25.5)$ & \\
\hline BMI distribution & & & & 0.485 \\
\hline$(\sim 18.4]$ & $25(5.2 \%)$ & $20(4.8 \%)$ & $5(8.1 \%)$ & \\
\hline$(18.5,23.9]$ & $271(56.1 \%)$ & $239(56.8 \%)$ & $32(51.6 \%)$ & \\
\hline$(24,27.9]$ & $155(32.1 \%)$ & $136(32.3 \%)$ & $19(30.6 \%)$ & \\
\hline$[28 \sim)$ & $32(6.6 \%)$ & $26(6.2 \%)$ & $6(9.7 \%)$ & \\
\hline Huanan seafood wholesale market exposure & & & & 0.574 \\
\hline No & $475(98.3 \%)$ & $413(98.1 \%)$ & $62(100 \%)$ & \\
\hline Yes & $8(1.7 \%)$ & $8(1.9 \%)$ & $0(0 \%)$ & \\
\hline History suspected patient exposure & & & & 0.080 \\
\hline Uncertain & $185(38.3 \%)$ & $168(39.9 \%)$ & $17(27.4 \%)$ & \\
\hline Yes & $298(61.7 \%)$ & $253(60.1 \%)$ & $45(72.6 \%)$ & \\
\hline With other family member infected & & & & 0.011 \\
\hline No & $263(54.5 \%)$ & $239(56.8 \%)$ & $24(38.7 \%)$ & \\
\hline Yes & $220(45.5 \%)$ & $182(43.2 \%)$ & $38(61.3 \%)$ & \\
\hline Daily exercise & & & & 0.730 \\
\hline No & $189(39.1 \%)$ & $163(38.7 \%)$ & $26(41.9 \%)$ & \\
\hline Yes & $294(60.9 \%)$ & $258(61.3 \%)$ & $36(58.1 \%)$ & \\
\hline Daily self-care ability & & & & $<0.001$ \\
\hline No & $22(4.6 \%)$ & $8(1.9 \%)$ & $14(22.6 \%)$ & \\
\hline Yes & $461(95.4 \%)$ & $413(98.1 \%)$ & $48(77.4 \%)$ & \\
\hline Smoking & & & & 0.063 \\
\hline Current smokers & $83(17.2 \%)$ & $66(15.7 \%)$ & $17(27.4 \%)$ & \\
\hline Give up smoking & $15(3.1 \%)$ & $14(3.3 \%)$ & $1(1.6 \%)$ & \\
\hline Never smokers & $385(79.7 \%)$ & $341(81.0 \%)$ & $44(71.0 \%)$ & \\
\hline Marital status & & & & 0.782 \\
\hline Divorce & $3(0.6 \%)$ & $3(0.7 \%)$ & $0(0 \%)$ & \\
\hline
\end{tabular}


Table 1 (continued)

\begin{tabular}{|c|c|c|c|c|}
\hline & Overall & Mild-moderate cases $(+/++)$ & Severe cases $(+++/++++)$ & $p^{\text {a value }}$ \\
\hline Married & $454(94.0 \%)$ & $395(93.8 \%)$ & $59(95.2 \%)$ & \\
\hline Unmarried & $26(5.4 \%)$ & $23(5.5 \%)$ & $3(4.8 \%)$ & \\
\hline Recent surgery history & & & & $<0.001$ \\
\hline No & $101(20.9 \%)$ & $99(23.5 \%)$ & $2(3.2 \%)$ & \\
\hline Yes & $382(79.1 \%)$ & $322(76.5 \%)$ & $60(96.8 \%)$ & \\
\hline Antiviral drug treatment before admission & & & & 0.874 \\
\hline No & $164(34.0 \%)$ & $144(34.2 \%)$ & $20(32.3 \%)$ & \\
\hline Yes & $319(66.0 \%)$ & $277(65.8 \%)$ & $42(67.7 \%)$ & \\
\hline Antibiotic drug treatment before admission & & & & 0.723 \\
\hline No & $193(40.0 \%)$ & $170(40.4 \%)$ & $23(37.1 \%)$ & \\
\hline Yes & $290(60.0 \%)$ & $251(59.6 \%)$ & $39(62.9 \%)$ & \\
\hline \multicolumn{5}{|l|}{ Comorbidities at admission } \\
\hline Primary pulmonary disease & & & & 0.047 \\
\hline No & $441(91.3 \%)$ & $389(92.4 \%)$ & $52(83.9 \%)$ & \\
\hline Yes & $42(8.7 \%)$ & $32(7.6 \%)$ & $10(16.1 \%)$ & \\
\hline Hypertension & & & & 0.067 \\
\hline No & $449(93.0 \%)$ & $392(93.1 \%)$ & $57(91.9 \%)$ & \\
\hline Yes & $34(7.0 \%)$ & $29(6.9 \%)$ & $5(8.1 \%)$ & \\
\hline Diabetes & & & & 0.072 \\
\hline No & $459(95.0 \%)$ & $401(95.2 \%)$ & $58(93.5 \%)$ & \\
\hline Yes & $24(5.0 \%)$ & $20(4.8 \%)$ & $4(6.5 \%)$ & \\
\hline Hyperlipidemia & & & & 0.124 \\
\hline No & $470(97.3 \%)$ & $412(97.9 \%)$ & $58(93.5 \%)$ & \\
\hline Yes & $13(2.7 \%)$ & $9(2.1 \%)$ & $4(6.5 \%)$ & \\
\hline Coronary heart disease & & & & $<0.001$ \\
\hline No & $475(98.3 \%)$ & $418(99.3 \%)$ & $57(91.9 \%)$ & \\
\hline Yes & $8(1.7 \%)$ & $3(0.7 \%)$ & $5(8.1 \%)$ & \\
\hline History of myocardial infarction & & & & 0.048 \\
\hline No & $476(98.6 \%)$ & $417(99.0 \%)$ & $59(95.2 \%)$ & \\
\hline Yes & $7(1.4 \%)$ & $4(1.0 \%)$ & $3(4.8 \%)$ & \\
\hline Cerebral infarction & & & & 0.849 \\
\hline No & $478(99.0 \%)$ & $416(98.8 \%)$ & $62(100 \%)$ & \\
\hline Yes & $5(1.0 \%)$ & $5(1.2 \%)$ & $0(0 \%)$ & \\
\hline Cerebral hemorrhage & & & & 0.266 \\
\hline No & $482(99.8 \%)$ & $420(99.8 \%)$ & $62(100 \%)$ & \\
\hline Yes & $1(0.2 \%)$ & $1(0.2 \%)$ & $0(0 \%)$ & \\
\hline Malignant neoplasms & & & & 0.849 \\
\hline No & $478(99.0 \%)$ & $416(98.8 \%)$ & $62(100 \%)$ & \\
\hline Yes & $5(1.0 \%)$ & $5(1.2 \%)$ & $0(0 \%)$ & \\
\hline Other disease & & & & 0.039 \\
\hline No & $442(91.5 \%)$ & $390(92.6 \%)$ & $52(83.9 \%)$ & \\
\hline Yes & $41(8.5 \%)$ & $31(7.4 \%)$ & $10(16.1 \%)$ & \\
\hline \multicolumn{5}{|l|}{ Vital signs at admission ${ }^{\mathrm{b}}$} \\
\hline Blood pressure & & & & 0.943 \\
\hline Hight blood pressure & $34(7.0 \%)$ & $29(6.9 \%)$ & $5(8.1 \%)$ & \\
\hline Normal blood pressure & $449(93.0 \%)$ & $392(93.1 \%)$ & $57(91.9 \%)$ & \\
\hline Breath & & & & $<0.001$ \\
\hline Breathing faster & $32(6.6 \%)$ & $20(4.8 \%)$ & $12(19.4 \%)$ & \\
\hline Normal breathing & $451(93.4 \%)$ & $401(95.2 \%)$ & $50(80.6 \%)$ & \\
\hline
\end{tabular}


Table 1 (continued)

\begin{tabular}{|c|c|c|c|c|}
\hline & Overall & Mild-moderate cases $(+/++)$ & Severe cases $(+++/++++)$ & $p^{\mathrm{a}}$ value \\
\hline Heart rate & & & & $<0.001$ \\
\hline Increased heart rate & $32(6.6 \%)$ & $21(5.0 \%)$ & $11(17.7 \%)$ & \\
\hline Normal heart rate & $451(93.4 \%)$ & $400(95.0 \%)$ & $51(82.3 \%)$ & \\
\hline Symptom at admission & & & & 0.897 \\
\hline Mild & $45(9.3 \%)$ & $39(9.3 \%)$ & $6(9.7 \%)$ & \\
\hline Moderate & $438(90.7 \%)$ & $382(90.7 \%)$ & $56(90.3 \%)$ & \\
\hline Highest temperature & & & & 0.029 \\
\hline Mean (SD, min, max) & $37.7(0.86,36.0,40.0)$ & $37.7(0.86,36.0,40.0)$ & $37.9(0.89,36.5,39.9)$ & \\
\hline Median (IQR) & $37.8(36.9,38.4)$ & $37.7(36.8,38.3)$ & $37.9(37.2,38.6)$ & \\
\hline Temperature distribution & & & & 0.195 \\
\hline$<37.5^{\circ} \mathrm{C}$ & $176(36.4 \%)$ & $158(37.5 \%)$ & $18(29.0 \%)$ & \\
\hline $37.5-38.0^{\circ} \mathrm{C}$ & $112(23.2 \%)$ & $97(23.0 \%)$ & $15(24.2 \%)$ & \\
\hline $38.1-39.0^{\circ} \mathrm{C}$ & $137(28.4 \%)$ & $113(26.8 \%)$ & $24(38.7 \%)$ & \\
\hline$>39.0^{\circ} \mathrm{C}$ & $58(12.0 \%)$ & $53(12.6 \%)$ & $5(8.1 \%)$ & \\
\hline Cough & & & & 0.524 \\
\hline No & $204(42.2 \%)$ & $175(41.6 \%)$ & $29(46.8 \%)$ & \\
\hline Yes & $279(57.8 \%)$ & $246(58.4 \%)$ & $33(53.2 \%)$ & \\
\hline Shortness of breath & & & & 0.041 \\
\hline No & $352(72.9 \%)$ & $314(74.6 \%)$ & $38(61.3 \%)$ & \\
\hline Yes & $131(27.1 \%)$ & $107(25.4 \%)$ & $24(38.7 \%)$ & \\
\hline Myalgia & & & & 0.439 \\
\hline No & $334(69.2 \%)$ & $288(68.4 \%)$ & $46(74.2 \%)$ & \\
\hline Yes & $149(30.8 \%)$ & $133(31.6 \%)$ & $16(25.8 \%)$ & \\
\hline Running nose & & & & 0.240 \\
\hline No & $408(84.5 \%)$ & $352(83.6 \%)$ & $56(90.3 \%)$ & \\
\hline Yes & $75(15.5 \%)$ & $69(16.4 \%)$ & $6(9.7 \%)$ & \\
\hline Arthralgia & & & & 0.263 \\
\hline No & $383(79.3 \%)$ & $330(78.4 \%)$ & $53(85.5 \%)$ & \\
\hline Yes & $100(20.7 \%)$ & $91(21.6 \%)$ & $9(14.5 \%)$ & \\
\hline Chest tightness & & & & 0.288 \\
\hline No & $365(75.6 \%)$ & $322(76.5 \%)$ & $43(69.4 \%)$ & \\
\hline Yes & $118(24.4 \%)$ & $99(23.5 \%)$ & $19(30.6 \%)$ & \\
\hline Nausea or vomiting & & & & 0.015 \\
\hline No & $415(85.9 \%)$ & $355(84.3 \%)$ & $60(96.8 \%)$ & \\
\hline Yes & $68(14.1 \%)$ & $66(15.7 \%)$ & $2(3.2 \%)$ & \\
\hline Headache & & & & 0.892 \\
\hline No & $381(78.9 \%)$ & $333(79.1 \%)$ & $48(77.4 \%)$ & \\
\hline Yes & $102(21.1 \%)$ & $88(20.9 \%)$ & $14(22.6 \%)$ & \\
\hline Fatigue & & & & 0.833 \\
\hline No & $461(95.4 \%)$ & $401(95.2 \%)$ & $60(96.8 \%)$ & \\
\hline Yes & $22(4.6 \%)$ & $20(4.8 \%)$ & $2(3.2 \%)$ & \\
\hline Pharyngalgia & & & & 0.606 \\
\hline No & $481(99.6 \%)$ & $419(99.5 \%)$ & $62(100 \%)$ & \\
\hline Yes & $2(0.4 \%)$ & $2(0.5 \%)$ & $0(0 \%)$ & \\
\hline Nasal congestion & & & & 0.606 \\
\hline No & $481(99.6 \%)$ & $419(99.5 \%)$ & $62(100 \%)$ & \\
\hline Yes & $2(0.4 \%)$ & $2(0.5 \%)$ & $0(0 \%)$ & \\
\hline Diarrhea & & & & 0.012 \\
\hline No & $447(92.5 \%)$ & $395(93.8 \%)$ & $52(83.9 \%)$ & \\
\hline
\end{tabular}


Table 1 (continued)

\begin{tabular}{|c|c|c|c|c|}
\hline & Overall & Mild-moderate cases $(+/++)$ & Severe cases $(+++/++++)$ & $p^{\mathrm{a}}$ value \\
\hline Yes & $36(7.5 \%)$ & $26(6.2 \%)$ & $10(16.1 \%)$ & \\
\hline \multicolumn{5}{|l|}{ Chill } \\
\hline No & $478(99.0 \%)$ & $417(99.0 \%)$ & $61(98.4 \%)$ & \\
\hline Yes & $5(1.0 \%)$ & $4(1.0 \%)$ & $1(1.6 \%)$ & \\
\hline \multicolumn{5}{|l|}{ Laboratory test results at admission ${ }^{\mathrm{c}}$} \\
\hline Leukocyte & & & & 0.025 \\
\hline Abnormal & $120(24.8 \%)$ & $97(23.0 \%)$ & $23(37.1 \%)$ & \\
\hline Normal & $363(75.2 \%)$ & $324(77.0 \%)$ & $39(62.9 \%)$ & \\
\hline Lymphocyte & & & & $<0.001$ \\
\hline Abnormal & $87(18.0 \%)$ & $64(15.2 \%)$ & $23(37.1 \%)$ & \\
\hline Normal & $396(82.0 \%)$ & $357(84.8 \%)$ & $39(62.9 \%)$ & \\
\hline Blood glucose & & & & 0.374 \\
\hline Abnormal glucose & $24(5.0 \%)$ & $19(4.5 \%)$ & $5(8.1 \%)$ & \\
\hline Normal glucose & $459(95.0 \%)$ & $402(95.5 \%)$ & $57(91.9 \%)$ & \\
\hline Renal function & & & & $<0.001$ \\
\hline Normal & $472(97.7 \%)$ & $420(99.8 \%)$ & $52(83.9 \%)$ & \\
\hline Abnormal & $11(2.3 \%)$ & $1(0.2 \%)$ & $10(16.1 \%)$ & \\
\hline Heart function & & & & $<0.001$ \\
\hline Normal & $466(96.5 \%)$ & $416(98.8 \%)$ & $50(80.6 \%)$ & \\
\hline Abnormal & $17(3.5 \%)$ & $5(1.2 \%)$ & $12(19.4 \%)$ & \\
\hline Liver function & & & & $<0.001$ \\
\hline Normal & $463(95.9 \%)$ & $413(98.1 \%)$ & $50(80.6 \%)$ & \\
\hline Abnormal & $20(4.1 \%)$ & $8(1.9 \%)$ & $12(19.4 \%)$ & \\
\hline Urine infection & & & & 0.129 \\
\hline No & $435(90.1 \%)$ & $383(91.0 \%)$ & $52(83.9 \%)$ & \\
\hline Yes & $48(9.9 \%)$ & $38(9.0 \%)$ & $10(16.1 \%)$ & \\
\hline Imaging of lung & & & & $<0.001$ \\
\hline Normal & $458(94.8 \%)$ & $415(98.6 \%)$ & $43(69.4 \%)$ & \\
\hline Abnormal & $25(5.2 \%)$ & $6(1.4 \%)$ & $19(30.6 \%)$ & \\
\hline Mental state before admission ${ }^{\mathrm{d}}$ & & & & 0.076 \\
\hline Nervous before admission & $166(34.4 \%)$ & $138(32.8 \%)$ & $28(45.2 \%)$ & \\
\hline Without nervous before admission & $317(65.6 \%)$ & $283(67.2 \%)$ & $34(54.8 \%)$ & \\
\hline Sleep quality since diagnosis & & & & 0.005 \\
\hline $\mathrm{Bad}$ & $123(25.5 \%)$ & $97(23.0 \%)$ & $26(41.9 \%)$ & \\
\hline Good & $20(4.1 \%)$ & $19(4.5 \%)$ & $1(1.6 \%)$ & \\
\hline Without influence & $340(70.4 \%)$ & $305(72.4 \%)$ & $35(56.5 \%)$ & \\
\hline
\end{tabular}

${ }^{\text {a }}$ Data are $n(\%)$ unless otherwise specified; $p$ values demonstrate differences between No conversion to severe and conversion to severe patients. $p<0.05$ was considered obviously significant

${ }^{\mathrm{b}}$ Hypertension, $\geq 140 / 90 \mathrm{mmHg}$; breath, 12-20 times/min; heart rate, 60-100 times/min

${ }^{\mathrm{c}}$ Normal reference value [1]: leukocyte: adult, $(4.0-10.0) \times 10^{\wedge} 9 / \mathrm{L}$; child, $(5.0-12.0) \times 10^{\wedge} 9 / \mathrm{L}$ [2]; lymphocyte percentage (Lymph\%) $20-40 \%$; lymphocyte absolute value (Lymph \#) 1.1-3.2 × 10^9 [3]; fasting whole blood glucose 3.9 6.1 mmol/L, $1 \mathrm{~h}$ after meal $6.7 \sim 9.4 \mathrm{mmol} / \mathrm{L}, 2 \mathrm{~h}$ after meal $\leq$ $7.8 \mathrm{mmol} / \mathrm{L}$

${ }^{\mathrm{d}}$ Heart function: tachycardia (100 beats/min)

${ }^{\mathrm{e}}$ Liver function: ALT 0-46 U/L; AST 0-46 U/L

${ }^{\mathrm{f}}$ Urine infection: creatinine $(30-110 \mathrm{umol} / \mathrm{L})$

progressed to critically ill status, suggesting that there may be individual differences in body temperature monitoring and even in the early concealment of the virus [8].
Consistent with the transmission route, we also found that critically ill patients were characterized by familial cluster infections, which indirectly confirms that COVID-19 can 
be transmitted through contact [9-11]. If necessary, appropriate psychological intervention during the admission of a patient may contribute to elevating the patient's condition.

So far, the COVID-19 infection has been managed by controlling the source of infection and cutting off the route of transmission dominates, but no effective treatment has been proposed. For critically ill patients, supportive treatments may continue for some time. According to our study, all the cases in the mobile cabin hospital were community-acquired viral infections; no cases of nosocomial infections were found. This also suggested that the safety isolation measures adopted by patients and medical workers in the mobile cabin hospital can significantly reduce the chance of cross-infection.

Several limitations should be highlighted. First, this was a retrospective study and inherent limitations existed; we tried our best to collect detailed information, but not all laboratory information were collected adequately. Second, 23 patients were lost to follow-up, including 10 of them who also refused our follow-up (for this part, the medical records suggested that they were alive), which enabled a lack of analyzing the outcome of patients after being transferred to designated hospital. These patients who lost to follow-up may have a certain impact on the results, especially deviations and existing biases, so exploring the potential risks associated with the deterioration of patients is infeasible. However, depending on this descriptive study, we found that severe cases were associated with comorbidities. We believe that our study population is representative of mild-moderate cases, especially those who transferred to severe cases, for which provided feasible tactics in management of COVID-19 infection.

Open Access This article is licensed under a Creative Commons Attribution 4.0 International License, which permits use, sharing, adaptation, distribution and reproduction in any medium or format, as long as you give appropriate credit to the original author(s) and the source, provide a link to the Creative Commons licence, and indicate if changes were made. The images or other third party material in this article are included in the article's Creative Commons licence, unless indicated otherwise in a credit line to the material. If material is not included in the article's Creative Commons licence and your intended use is not permitted by statutory regulation or exceeds the permitted use, you will need to obtain permission directly from the copyright holder. To view a copy of this licence, visit http://creativecommons.org/licenses/by/4.0/.

\section{References}

1. World Health Organization. Novel coronavirus(2019-nCoV): situation report-15. February 5, 2020. https://www.who.int/docs/ default-source/coronaviruse/situation-reports/20200204-sitrep-15ncov.pdf

2. Holshue ML, DeBolt C, Lindquist S et al First Case of 2019 Novel Coronavirus in the United States. N Engl J Med:2020

3. National Health Committee of the People's Republic of China. New coronavirus pneumonia diagnosis and treatment plan. February 18 , 2020. http://www.nhc.gov.cn/wjw/gfxwjj/list.shtml

4. Chen N, Zhou M, Dong X et al (2020) Epidemiological and clinical characteristics of 99 cases of 2019 novel coronavirus pneumonia in Wuhan, China: a descriptive study. Lancet 395(10223):507-513

5. Huang C, Wang Y, Li X et al (2020) Clinical features of patients infected with 2019 novel coronavirus in Wuhan, China. Lancet 395(10223):497-506

6. Wang D, Hu B, Hu C et al (2020) Clinical characteristics of 138 hospitalized patients with 2019 novel coronavirus-infected pneumonia in Wuhan, China. JAMA

7. Yang X, Yu Y, Xu J et al (2020) Clinical course and outcomes of critically ill patients with SARS-CoV-2 pneumonia in Wuhan, China: a single-centered, retrospective, observational study. Lancet Respir Med

8. Guan W-j, Ni Z-y, Hu Y et al (2020) Clinical Characteristics of Coronavirus Disease 2019 in China. N Engl J Med

9. Zhu N, Zhang D, Wang W et al (2020) A novel coronavirus from patients with pneumonia in China, 2019. N Engl J Med 382(8): 727-733

10. Bajema KL, Oster AM, McGovern OL et al (2020) Persons evaluated for 2019 novel coronavirus - United States, January 2020. MMWR Morb Mortal Wkly Rep 69(6):166-170

11. Chan JF-W, Yuan S, Kok K-H et al (2020) A familial cluster of pneumonia associated with the 2019 novel coronavirus indicating person-to-person transmission: a study of a family cluster. Lancet 395(10223):514-523

Publisher's note Springer Nature remains neutral with regard to jurisdictional claims in published maps and institutional affiliations. 